\title{
Spherical collapse of fuzzy dark matter
}

\author{
V. Sreenath ${ }^{1, *}$ \\ ${ }^{1}$ Inter-University Centre for Astronomy and Astrophysics, \\ Post Bag 4, Ganeshkhind, Pune 41100\%, India.
}

It has been postulated that Fuzzy Dark Matter (FDM) could be a viable alternative to Cold Dark Matter (CDM). FDM is comprised of ultralight bosons which exist as a BoseEinstein condensate. Due to the very low mass of FDM, the de Broglie wavelength of these bosons are of the order of $\mathrm{kpc}$ and the quantum effects manifest at those scales. Hence, unlike CDM, FDM experiences quantum pressure along with gravitational attraction. In this work, we investigate the gravitational collapse of a spherically symmetric FDM overdensity. We assume a power law density profile for an overdense region of FDM and derive an expression for the temporal evolution of a spherical shell in the non-interacting limit and use it to derive an expression for average overdensity contained in the spherical shell in an Einsteinde Sitter universe. Further, we numerically extend the analysis to the case of interacting bosons. Finally, we discuss the virialization of such an overdense region of non-interacting FDM and derive an expression for overdensity in the linear and the full theory. We compare our results with those obtained in the case of CDM and conclude with a discussion of the results.

\footnotetext{
*vsreenath@iucaa.in
} 


\section{INTRODUCTION}

Standard model of cosmology, namely the $\Lambda$ CDM model has been a grand success [1]. However this success also poses some serious questions. Of them a chief concern is regarding the nature of dark matter. Despite the success of Cold Dark Matter(CDM) at large scales, it has met with some problems at scales less than $10 \mathrm{kpc}$ (for a recent review, see, for instance, [2]). CDM predicts [3] that the halos have a cusp in the density profile at its center. However, observations [4-6] of low surface brightness galaxies and dwarf galaxies indicate that the density profile at the center of halos are shallower or in other words has a core (for a review, see, for instance, [7]). Furthermore, simulations of CDM over predicts the number of dwarf galaxies in the local group by an order of magnitude [8]. Of these two difficulties faced by CDM, it has been suggested that, the latter may be alleviated to an extent by taking in to account the effects due to baryons (for a recent work, see, for instance, [9]). However, the dust is yet to settle.

In order to overcome the small scale issues of CDM, several alternatives to CDM has been suggested. One such alternative is Warm Dark Matter (WDM) (see, for instance, [10]). In this model, the dark matter particles possess a thermal velocity which causes them to free stream. This free streaming suppresses the formation of small scale structures thus solving the over abundance of dwarf galaxies and the core-cusp problem [11, 12]. However, the free streaming may also lead to certain imprints at large scale which can only be fixed by fine-tuning the parameters[13]. Another variant of CDM is the collisional dark matter (for a recent review, see, for instance, [14]). It has been shown that the presence of collisions flattens the core and destroys the dwarf galaxies [15]. However, an excess amount of collisions could also lead to the formation of singular core [16].

Another proposal, which we will concern ourselves with in this article, is that the dark matter is composed of ultralight bosons [17]. A popular candidate of such an ultralight bosonic dark matter, known commonly as Fuzzy Dark Matter (FDM), is axion of mass $m \sim 10^{-22}-10^{-21}$ $\mathrm{eV}$ (for reviews on axion cosmology, see $[18,19]$ ). All large scale properties of FDM is similar to that of CDM. However, at small scales quantum properties of FDM affects the formation of structure. Due to the small mass of FDM, the de Broglie wavelength is of order of kpc. The de Broglie wavelength manifests itself as a Jeans length below which the quantum pressure due to the uncertainty principle acts against gravity. Thus, below the de Broglie wavelength, the pressure suppresses the formation of structure and flattens the density profile[17, 20-22]. The implications of FDM model to structure formation has been investigated (see, for instance, [23, 24]). Most of the current searches of dark matter are not designed to detect FDM and hence the negative results do not constrain it. However, experiments have been proposed which are likely to detect FDM [25-31].

In the FDM model, the ultralight bosons form a Bose-Einstein Condensate (BEC) at a very early time. In a BEC all the dark matter particles occupy the ground state and hence is described by a coherent wave function. The evolution of such a system is described by the Schrödinger equation together with an equation governing gravity. The exact dynamics of structure formation can only be explored using numerical simulations [32-35]. High-resolution numerical simulations [33] show that the halo centers have a solitonic core with the outer profile similar to NFW [3]. Even though numerical simulations are required to have a complete understanding of the structure formation, analytical approximations often give useful insights. In this spirit, there has been analytical investigations of the steady state of a spherically symmetric Newtonian self-gravitating BEC [36-42]. In these studies, the nature of the virialized halo has been investigated, either assuming that the system is in hydrostatic equilibrium or by using virial theorem. For some other approaches to the study of collapse of axionic scalar field or formation of structure from axions, see, for instance, [43-49]. In this work, following the footsteps of an earlier work on CDM [50], we would like to study the gravitational collapse of a spherically overdense region of FDM. Firstly, assuming a 
spherically symmetric power law density profile, we will analytically investigate the time evolution of a spherical shell of FDM comprising of non-interacting bosons. We will use the analytical solutions for radius of the shell to arrive at an expression for overdensity and study it in the linear regime. Secondly, we will extend the analysis numerically to the case of interacting bosons, and study the evolution of a spherical shell and its dependence on the strength of interactions. Finally, we will use virial theorem for non-interacting FDM to compute the critical overdensity at which the spherically overdense region would collapse in to a halo.

The rest of the paper is organized as follows. In the next section we will describe the GrossPitaevskii-Poisson system that governs a BEC evolving under the effect of gravity. We describe how such a system can be expressed as hydrodynamic equations, namely the continuity, Euler and Poisson equations. From the hydrodynamic equations, we derive the equation of motion of a spherical shell containing an overdense region of FDM. In section III, assuming a density profile for the overdense region, we analytically and numerically solve for the equation of motion of the spherical shell to calculate the evolution of the shell with time and use the analytical expression to compute the expression for averaged overdensity contained in the spherical shell as a function of time. We further compute the evolution of the shell, numerically, for the case of interacting bosons. In section IV, we use virial theorem to compute the critical overdensity at which the overdense region will collapse in to a halo. We conclude the paper in section $\mathrm{V}$ with a discussion of the results.

We shall work with the units where $c=1$.

\section{GROSS-PITAEVSKII-POISSON SYSTEM AND EQUATION FOR SPHERICAL COLLAPSE}

FDM is a BEC evolving under the effect of gravity. Since, general relativistic effects are not significant at the scale of a halo we can use Newtonian gravity. The state of such a system is described by the condensate wave function, $\psi(t, \vec{r})$, governed by the Gross-Pitaevskii-Poisson (GPP) equations (see, for instance, $[51,52]$ ),

$$
\begin{aligned}
i \hbar \frac{\partial \psi(t, \vec{r})}{\partial t} & =-\frac{\hbar^{2}}{2 m} \nabla^{2} \psi(t, \vec{r})+m \Phi(t, \vec{r}) \psi(t, \vec{r})+\frac{4 \pi a_{s} \hbar^{2}}{m^{2}}|\psi(t, \vec{r})|^{2} \psi(t, \vec{r}) \\
\nabla^{2} \Phi(t, \vec{r}) & =4 \pi \mathrm{G}|\psi(t, \vec{r})|^{2},
\end{aligned}
$$

where $m$ is the mass of the boson, $\rho(t, \vec{r})=|\psi(t, \vec{r})|^{2}$ is the mass density and $\Phi(t, \vec{r})$ is the gravitational potential. The self-interaction of bosons is described by the s-wave scattering length, $a_{s}$, which can be positive (repulsive), negative (attractive) or zero (non-interacting). For brevity, whenever possible, we will not explicitly write the coordinate dependence of quantities.

\section{A. Hydrodynamic equations}

It is often convenient to express the GPP equations, describing the FDM halo, in terms of fluid variables, namely density and velocity [52]. This can be achieved by performing a Madelung transformation [53],

$$
\psi(t, \vec{r})=\sqrt{\rho(t, \vec{r})} \exp (i S(t, \vec{r}) / \hbar)
$$

Upon substituting Eq. (2.2) in Eq. (2.1a), defining

$$
\vec{u}(t, \vec{r}) \equiv \frac{\vec{\nabla} S(t, \vec{r})}{m}
$$


equating real and imaginary parts of Eq. (2.1a) and using the identity

$$
(\vec{u} \cdot \vec{\nabla}) \vec{u}=\vec{\nabla}\left(u^{2} / 2\right)-\vec{u} \times(\vec{\nabla} \times \vec{u})=\vec{\nabla}\left(u^{2} / 2\right),
$$

we obtain

$$
\begin{aligned}
\frac{\partial \rho}{\partial t}+\vec{\nabla} \cdot(\rho \vec{u}) & =0, \\
\frac{\partial \vec{u}}{\partial t}+(\vec{u} \cdot \vec{\nabla}) \vec{u} & =-\frac{\vec{\nabla} P}{\rho}-\vec{\nabla} \Phi-\frac{\vec{\nabla} Q}{m}, \\
\nabla^{2} \Phi & =4 \pi \mathrm{G} \rho,
\end{aligned}
$$

which are, respectively, the continuity, Euler and Poisson equations of a fluid with density $\rho$ and velocity $\vec{u}$. Equation (2.3) implies that the velocity, $\vec{u}$, of the fluid is irrotational. In the Euler equation, we have denoted the quantum potential by

$$
Q(t, \vec{r})=-\frac{\hbar^{2}}{2 m} \frac{\nabla^{2} \sqrt{\rho}}{\sqrt{\rho}}=-\frac{\hbar^{2}}{4 m}\left[\frac{\nabla^{2} \rho}{\rho}-\frac{1}{2} \frac{(\nabla \rho)^{2}}{\rho^{2}}\right]
$$

and the pressure, arising due to self interactions, by

$$
P(t, \vec{r})=\frac{2 \pi a_{s} \hbar^{2}}{m^{3}} \rho^{2} .
$$

Note that the above equation describes an equation of state of a polytrope of index one.

In an expanding universe, $\vec{r}(t)=a(t) \vec{x}$. Using the relation,

$$
\left.\frac{\partial}{\partial t}\right|_{\vec{r}}=\left.\frac{\partial}{\partial t}\right|_{\vec{x}}-H \vec{x} \cdot \vec{\nabla},
$$

where $H(t)=\dot{a}(t) / a(t)$ is the Hubble parameter, the fluid equations can be written as

$$
\begin{aligned}
& \frac{\partial \rho}{\partial t}-H(\vec{x} \cdot \vec{\nabla}) \rho+\frac{\vec{\nabla} \cdot(\rho \vec{u})}{a}=0, \\
& \frac{\partial \vec{u}}{\partial t}-H(\vec{x} \cdot \vec{\nabla}) \vec{u}+\frac{(\vec{u} \cdot \vec{\nabla}) \vec{u}}{a}=-\frac{\vec{\nabla} P}{a \rho}-\frac{\vec{\nabla} \Phi}{a}-\frac{\vec{\nabla} Q}{a m}, \\
& \nabla^{2} \Phi=4 \pi \mathrm{G} a^{2} \rho,
\end{aligned}
$$

where $\vec{\nabla}$ is now with respect to $\vec{x}$,

Let us now separate the density of the condensate in to a background part and a perturbation on top of it, i.e. $\rho=\rho_{b}(1+\delta)$, where $\delta=\delta \rho / \rho_{b}$. A similar split can be made to the velocity of the fluid element in to the Hubble flow and peculiar velocity, i.e. $\vec{u}=H \vec{r}+\vec{v}$, respectively. Using these definitions, one could write the perturbed part of the fluid equations as

$$
\begin{aligned}
& \frac{\partial \delta}{\partial t}+\frac{\vec{\nabla}}{a} \cdot[\vec{v}(1+\delta)]=0 \\
& \frac{\partial \vec{v}}{\partial t}+H \vec{v}+\frac{(\vec{v} \cdot \vec{\nabla}) \vec{v}}{a}=-\frac{4 \pi a_{s} \hbar^{2}}{a m^{3}} \vec{\nabla} \rho-\frac{\vec{\nabla} \Phi_{p}}{a}+\frac{\hbar^{2}}{4 m^{2} a^{3}} \vec{\nabla}\left[\frac{\nabla^{2} \rho}{\rho}-\frac{1}{2} \frac{(\nabla \rho)^{2}}{\rho^{2}}\right] \\
& \nabla^{2} \Phi_{p}=4 \pi \mathrm{G} a^{2} \rho_{b} \delta
\end{aligned}
$$

In the above, we have also divided the gravitational potential, $\Phi=\Phi_{b}+\Phi_{p}$, in to background and perturbation parts respectively. It can be shown that $\Phi_{b}=-\ddot{a} r^{2} /(2 a)$. In writing the perturbed part of Euler equation, we have retained the full density, $\rho$, on the right-hand side for later convenience. 


\section{B. Equation for a collapsing spherical shell}

Consider a spherically overdense distribution of FDM. We are interested in understanding the evolution of such an overdense region with time. Consider a spherical shell of radius $R(t)=$ $a(t) X(t)$, enclosing certain mass, centered in the overdense region. A fluid element on that shell would have a velocity, $\vec{u}=H \vec{R}+\vec{v}$, where the velocity of the fluid element is in radial direction. The acceleration of that fluid element can be computed as

$$
\frac{\mathrm{d}^{2} \vec{R}}{\mathrm{~d} t^{2}}=\frac{\mathrm{d} \vec{u}}{\mathrm{~d} t}=\dot{H} \vec{R}+H(H \vec{R}+\vec{v})+\frac{\partial \vec{v}}{\partial t}+\frac{(\vec{v} \cdot \vec{\nabla})}{a} \vec{v} .
$$

Upon using the Euler equation, Eq. (2.14), and the fact that $\vec{\nabla} \Phi_{b}=-\ddot{a} \vec{R} / a^{2}$, we obtain

$$
\frac{\mathrm{d}^{2} \vec{R}}{\mathrm{~d} t^{2}}=-\frac{\vec{\nabla} \Phi_{b}}{a}-\frac{4 \pi a_{s} \hbar^{2}}{a m^{3}} \vec{\nabla} \rho-\frac{\vec{\nabla} \Phi_{p}}{a}+\frac{\hbar^{2}}{4 m^{2} a^{3}} \vec{\nabla}\left[\frac{\nabla^{2} \rho}{\rho}-\frac{1}{2} \frac{(\nabla \rho)^{2}}{\rho^{2}}\right] .
$$

Combining the background and perturbed parts of the gravitational potential, one can write the equation of motion of the spherical shell as

$$
\frac{\mathrm{d}^{2} \vec{R}}{\mathrm{~d} t^{2}}=-\frac{4 \pi a_{s} \hbar^{2}}{m^{3}} \vec{\nabla} \rho-\vec{\nabla} \Phi+\frac{\hbar^{2}}{4 m^{2}} \vec{\nabla}\left[\frac{\nabla^{2} \rho}{\rho}-\frac{1}{2} \frac{(\nabla \rho)^{2}}{\rho^{2}}\right],
$$

where the spatial derivatives are now with respect to $r$ and are evaluated on the shell, $r=R(t)$. Thus, the motion of the spherical shell is governed by a force arising due to the bosonic interactions which could be attractive $\left(a_{s}<0\right)$ or repulsive $\left(a_{s}>0\right)$, gravitational attraction and a quantum repulsive force. It can be seen that, in the limit $\hbar / m \rightarrow 0$, we reproduce the equation for spherical collapse of CDM. In order to make further progress, we need to assume a density profile and study the evolution of the above equation.

\section{SPHERICAL COLLAPSE OF FDM WITH A POWER LAW DENSITY PROFILE}

For the overdense region, we consider a power law density profile of the form

$$
\rho(t, r)=\frac{3-\gamma}{4 \pi} \frac{M}{L(t)^{3}}\left(\frac{r}{L(t)}\right)^{-\gamma}
$$

where the normalization factors has been chosen in such a way that, $L(t)$ is the radius of the shell which encloses a mass $M$ and $\gamma$ is a positive number less than 3 (by demanding that density should be positive). Assuming that the FDM overdense region maintains such a density profile throughout the evolution, one can derive the equation of motion for the spherical shell. For a shell of radius $L(t)$, containing mass $M$, the equation of motion can be written as

$$
\frac{\mathrm{d}^{2} L}{\mathrm{~d} t^{2}}=\gamma(3-\gamma) \frac{a_{s} \hbar^{2}}{m^{3}} \frac{M}{L^{4}}-\frac{\mathrm{G} M}{L^{2}}+\gamma(2-\gamma) \frac{\hbar^{2}}{4 m^{2} L^{3}}
$$

As explained before, the evolution of the shell is governed by three forces, namely, (i) the repulsive $\left(a_{s}>0\right)$ or attractive $\left(a_{s}<0\right)$ force due to bosonic self-interaction, (ii) attractive gravitational force and (iii) repulsive quantum force. Note that, for the power law profile, in order for the quantum force to be positive and non-vanishing, one requires $\gamma<2$. 


\section{A. Non-interacting bosons}

We will investigate the case of non-interacting bosons in this subsection and consider the effects of interaction in the next.

\section{Analytical solution}

In the absence of interactions $\left(a_{s}=0\right)$, the equation of motion of the spherical shell can be written as,

$$
m \frac{\mathrm{d}^{2} L}{\mathrm{~d} t^{2}}=-\frac{k}{L^{2}}+\frac{l^{2}}{m L^{3}}
$$

where $k=\mathrm{G} M m$ and $l^{2}=\left(2 \gamma-\gamma^{2}\right) \hbar^{2} / 4$. This equation is mathematically, though not physically, similar to the equation governing the reduced mass in a two-body Kepler problem (see, for instance, [54]). Hence, we will draw insights from the solution of Kepler problem to solve Eq. $(3.3)$.

Initially, let the overdense shell be expanding along with the Hubble flow. The shell will eventually turn around if the initial value of the first integral of motion of the shell is negative, i.e. if,

$$
E=\frac{1}{2} m\left(\frac{\mathrm{d} L}{\mathrm{~d} t}\right)^{2}+\frac{l^{2}}{2 m L^{2}}-\frac{k}{L}<0 .
$$

In such a case, it can be shown that the solution to Eq. (3.3) can be expressed as follows

$$
\begin{aligned}
L & =A(1-e \cos \vartheta), \\
t & =\left(\frac{m A^{3}}{k}\right)^{1 / 2}(\vartheta-e \sin \vartheta),
\end{aligned}
$$

where, $A=-k /(2 E)$ and the expression for $e$ is

$$
e=\sqrt{1+\frac{2 E l^{2}}{m k^{2}}}=\sqrt{1+\frac{E \hbar^{2}}{\mathrm{G}^{2} M^{2} m^{3}} \frac{\left(2 \gamma-\gamma^{2}\right)}{2}} .
$$

Note that, since $E<0$, the value of $e<1$. The Eqs. (3.5) can be combined to obtain the behaviour of $L$ as a function of $t$.

Let us now try to understand the behaviour of the solution. When $\vartheta=0, L(0)=L_{\text {min }}=$ $A(1-e)$ and when $\vartheta=\pi, L(\pi)=L_{\max }=A(1+e)$. Since $e<1$, the radius of the shell is thus bounded from below and hence will oscillate between the two extremum values.

\section{Numerical solution}

Let us now solve Eq. (3.3) numerically and compare it with the analytical solutions Eqs. (3.5). For numerical simulations, it is convenient to rewrite Eq. (3.3) in terms of dimensionless variables as,

$$
\frac{\mathrm{d}^{2} y}{\mathrm{~d} \tau^{2}}=\frac{2 \gamma-\gamma^{2}}{4 y^{3}}-\frac{1}{y^{2}}
$$

where, we have defined $y=L / L_{Q}$ and $\tau=t / t_{Q}$, with $L_{Q}=\hbar^{2} /\left(G M m^{2}\right)$ and $t_{Q}=\sqrt{L_{Q}^{3} /(G M)}$. In terms of $y$ and $\tau$, we only need to solve the differential equation for a given initial condition 


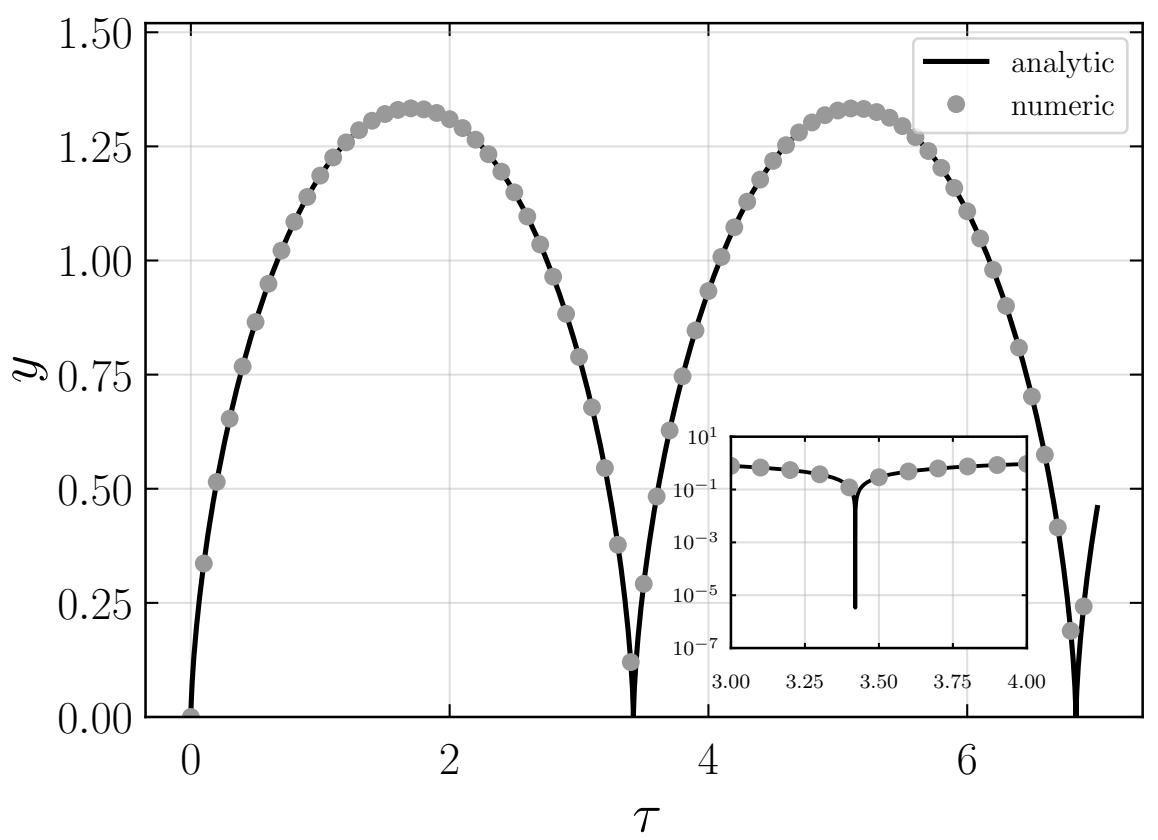

FIG. 1. Comparison of analytical and numerical results for the evolution of $y$ as a function of $\tau$. As expected, the numerical and analytical results match very well. For a shell containing a mass $M=9.1 \times 10^{7} \mathrm{M}_{\text {sun }}$ and for bosonic mass $m=8.1 \times 10^{-23} \mathrm{eV}$, this evolution corresponds to a shell oscillating between a minimum radius of $L_{\min }=3.57 \times 10^{-8} \mathrm{pc}$ and a maximum radius of $L_{\max }=1.9 \mathrm{kpc}$.

once. The solution for the shell with radius $L(t)$ containing any mass $M$ can be then obtained from solution for $y(\tau)$ by scaling it with corresponding $L_{Q}$ and $t_{Q}$.

In order to solve for $y(\tau)$, we assume that, in the beginning, the shell containing an average overdensity of $\bar{\delta}_{i}=10^{-5}$, is expanding according to the Hubble flow. Furthermore, we assume that the universe is Einstein-de Sitter(EdS); i.e. the scale factor scales with time as $a \propto t^{2 / 3}$. Since we are interested in bound solutions, we assume that the first integral of motion of Eq. (3.7) is less than zero. Using these three conditions, we can fix the initial conditions for Eq. (3.7). For $\gamma=10^{-10}$, we have numerically solved for $y(\tau)$ using Mathematica [55] and compared with the analytical solutions expressed in terms of $y(\tau)$. As can be seen from Fig. 1, as expected, both the solutions match very well. In order to make sense of the numbers involved, let us assume that the shell contains a mass of $M=9.1 \times 10^{7} \mathrm{M}_{\text {sun }}$ and that the mass of boson is $m=8.1 \times 10^{-23} \mathrm{eV}$ (values arrived at for Fornax dwarf spheroidal in [33]). Such a shell, for the initial condition we have considered, would oscillate between a minimum radius of $L_{\min }=3.57 \times 10^{-8} \mathrm{pc}$ and a maximum radius of $L_{\max }=1.9 \mathrm{kpc}$. We have considered an initial state for which $y_{i}=10^{-5}$ and $\bar{\delta}_{i}=10^{-5}$. For this state, it is interesting to note that $1-e=\mathcal{O}\left(10^{-11}\right)$, hence such a vast difference in $L_{\min }$ and $L_{\max }$.

\section{Overdensity}

Let us assume that the background spacetime is EdS. The average density contained in a spherical shell of radius $L$ containing mass $M$ is given by $\bar{\rho}=M /\left(4 \pi L^{3} / 3\right)$. In an EdS universe, the background density is given by $\rho_{b}=1 /\left(6 \pi \mathrm{G} t^{2}\right)$. Hence, the average overdensity inside the 
spherical shell is

$$
1+\bar{\delta}=\frac{\bar{\rho}}{\rho_{b}}=\frac{9}{2} \frac{G M t^{2}}{L^{3}}
$$

Substituting Eqs. (3.5) for $L$ and $t$, we obtain

$$
1+\bar{\delta}=\frac{9}{2} \frac{(\vartheta-e \sin \vartheta)^{2}}{(1-e \cos \vartheta)^{3}}
$$

The above expression for average overdensity within the shell has the following properties: (i) since $e<1$, the overdensity does not diverge as $\vartheta \rightarrow 2 \pi$, (ii) the averaged overdensity is fluctuating and increasing with time, (iii) in the limit $\hbar / m \rightarrow 0, e \rightarrow 1$, Eq. (3.9) reproduces the CDM expression (see, for instance, [56]) for averaged overdensity.

Let us now turn our attention to the behaviour of $\bar{\delta}$ in the small $\vartheta$ limit. Upon Taylor expanding the expression for $\bar{\delta}(\vartheta)$, Eq. (3.9), about $\vartheta \simeq 0$, we obtain

$$
1+\bar{\delta} \simeq \frac{9}{2}\left(\frac{\vartheta^{2}}{1-e}\right)-\frac{21 e}{4}\left(\frac{\vartheta^{2}}{1-e}\right)^{2}+\ldots
$$

The above expansion for $\bar{\delta}$ would be valid only if $\vartheta^{2}<<1-e$. However, in this limit, the above expression imply that $\bar{\delta} \simeq-1$ which indicate an underdensity. If as we saw in the last section, $1-e$ is very small, then one could first take the limit of $e \rightarrow 1$ and then the limit $\vartheta \rightarrow 0$. Upon taking the limit in this order, of Eqs. (3.5b) and (3.9), we obtain,

$$
\bar{\delta} \simeq \frac{3 \vartheta^{2}}{20} \propto a,
$$

which is similar to that in CDM. The above discussion seem to indicate that, in this model, for an overdense region, a sensible small $\vartheta$ limit exists only if the limit $e \rightarrow 1$ can be taken before the $\vartheta \rightarrow 0$ limit.

\section{B. Effect of interactions}

We shall now try to understand the effect of interactions. Due to the lack of analytical solution, we will approach the problem numerically. For $a_{s} \neq 0$, we can rewrite Eq. (3.2) in dimensionless form as

$$
\frac{\mathrm{d}^{2} y}{\mathrm{~d} \tau^{2}}=\frac{\alpha\left(3 \gamma-\gamma^{2}\right)}{y^{4}}+\frac{2 \gamma-\gamma^{2}}{4 y^{3}}-\frac{1}{y^{2}}
$$

where, $a_{s} \equiv \alpha \bar{a}_{s}$ with $\bar{a}_{s}=\hbar^{2} /\left(G M^{2} m\right)$ and $\alpha$ can be greater than, equal to or less than zero which corresponds to repulsive, nil and attractive interaction respectively. In order to understand the effect of interactions, it is convenient to look at the form of the effective potential governing the evolution of the shell,

$$
V(y)=\frac{\alpha\left(3 \gamma-\gamma^{2}\right)}{3 y^{3}}+\frac{2 \gamma-\gamma^{2}}{8 y^{3}}-\frac{1}{y}
$$

As one can see from Fig. 2, the effect of non-zero interaction adds up with the quantum pressure when $\alpha>0$, whereas, it acts against quantum pressure for $\alpha<0$. We can see that for

$\alpha \lesssim-1.6 \times 10^{-12}$, the attractive force due to interaction and gravity is stronger than the repulsive 


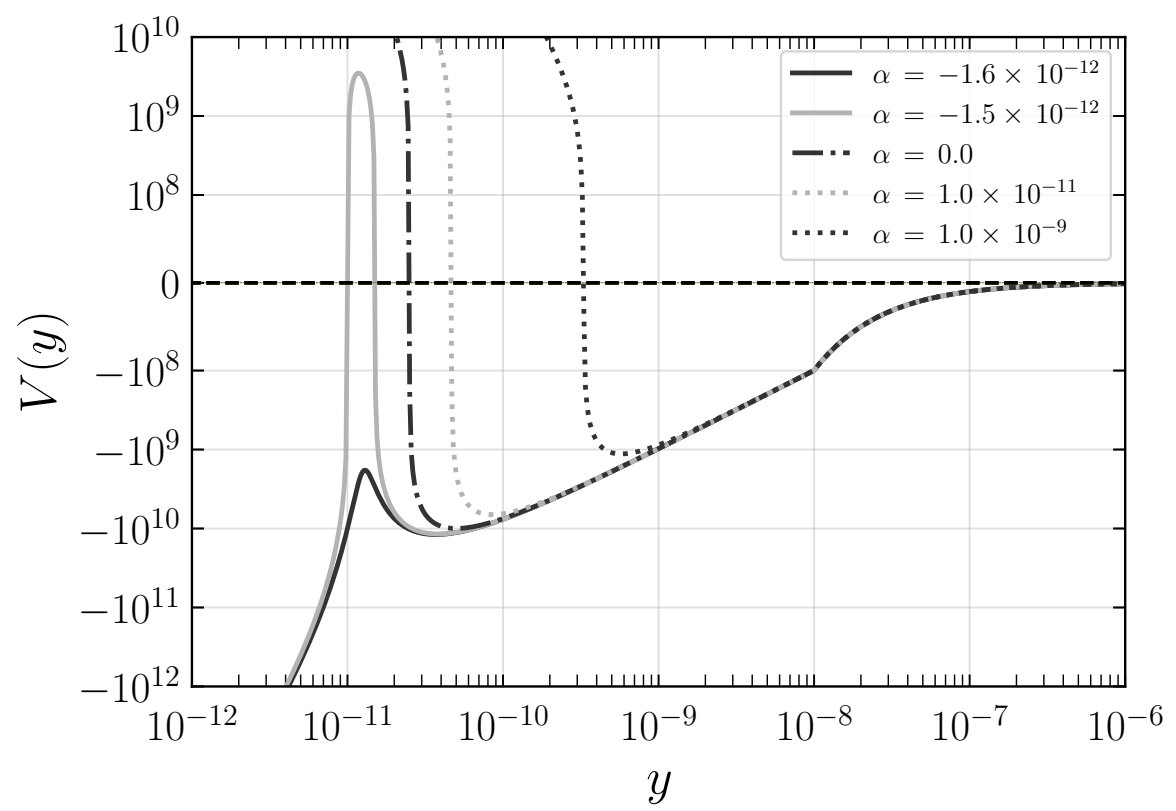

FIG. 2. Behaviour of effective potential as a function of $y$ for different values of $\alpha$. Horizontal black dashed line denotes the effective energy of the fluid element of the shell with a density profile specified by $\gamma=10^{-10}$ and with initial conditions $\bar{\delta}_{i}=10^{-5}$ and $y_{i}=10^{-5}$ and curves denote the effective potential of the fluid element for various values of $\alpha$. As we can see, for $\alpha=-1.6 \times 10^{-12}$, the potential does not have a region which is bounded from both sides and hence the quantum pressure cannot stop the collapse of the shell. For all other values of $\alpha$, shown in the figure, the shell will oscillate.

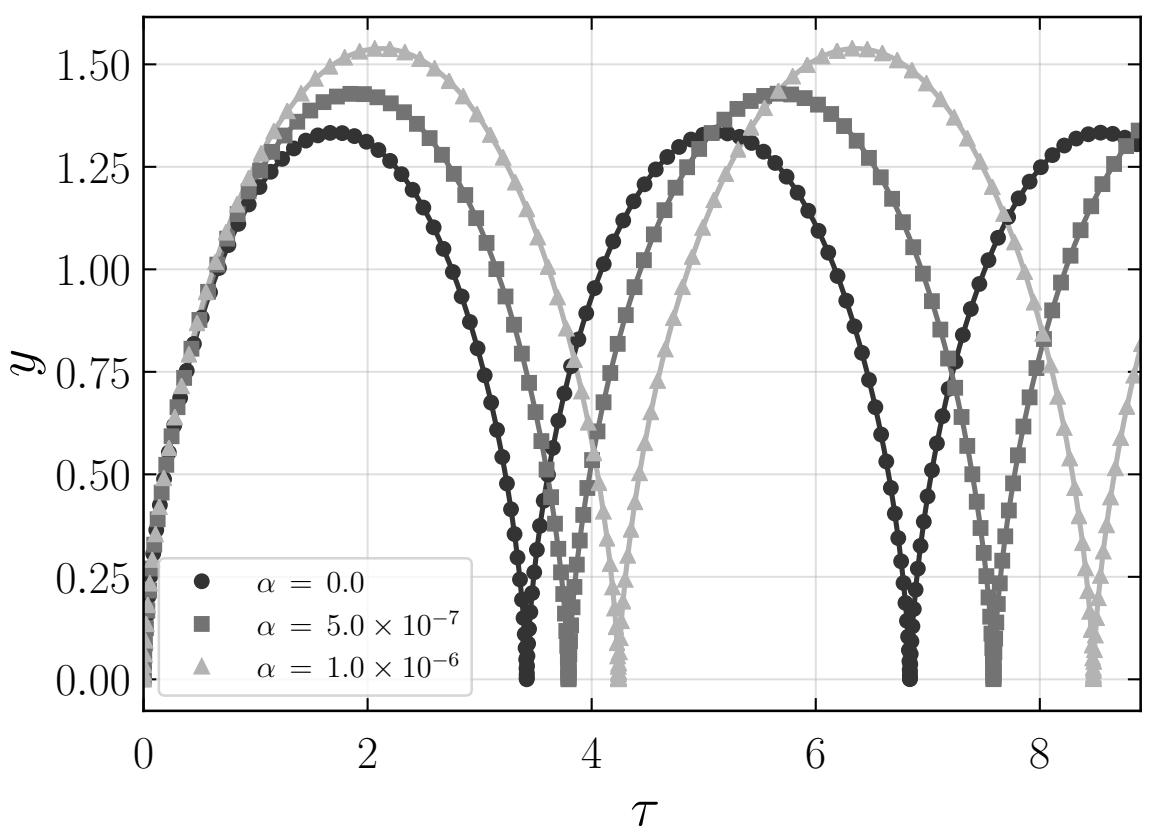

FIG. 3. The evolution of the shell for different values of $\alpha>0$ are shown. The effect of increasing $\alpha$ is a shift in the minimum of the potential to larger values of $y$. This would cause the fluid element to oscillate between larger values of maximum and minimum and with a longer period. The markers indicate the analytical expression Eq. (3.5). 
force due to quantum pressure and hence the shell will collapse. Thus, the existence of a core will allow us to put a lower bound on $a_{s}$ for attractive interactions. For the parameters that we have considered, we will have a lower bound of $a_{s} \gtrsim-5.3 \times 10^{-89} \mathrm{~m}$. In other words, for attractive scattering length, $\left|a_{s}\right|<<\bar{a}_{s}$ in order to sustain oscillations and hence form a core.

For $\alpha>0$, the effect of interactions is to push the minima of the potential to larger $y$. Hence, for larger $\alpha$, the shell would oscillate between larger maximum and minimum radius. In Fig. 3, we have plotted the numerical evolution of the shell for various values of $\alpha$. The dots indicate the analytic expression Eq. (3.5). For the parameters that we use, the match is apparently good. This is because, in this toy model, the effect of interactions is only felt at small scales where as at large scales, the force is dominated by gravity. Though not evident from the plot, the minimum value of $y$ differs from the analytical value as the value of $\alpha$ is non-zero. In particular, the analytical expression predicts a minimum radius of $y_{\min }=2.5 \times 10^{-11}$ where as the numerical simulations indicate a minimum radius of $1.5 \times 10^{-11}, 7.08 \times 10^{-9}$ and $1.0 \times 10^{-8}$ for $\alpha=-1.5 \times 10^{-12}, 5 \times 10^{-7}$ and $10^{-6}$ respectively. Hence, one can conclude that, for the parameters that we have considered, the analytical expression derived in Section III A 1 holds at large to medium scales for the case of interacting bosons.

\section{VIRIALIZATION}

The study of evolution of a single shell of radius $L$ of FDM containing a mass $M$ shows that a sufficiently overdense shell would initially expand along with the Hubble flow and like in the case of CDM turn around and start contracting. However, unlike CDM, instead of contracting to zero size, the shell may start expanding again and repeat the process of expansion, turn around and contraction. For the case of non-interacting bosons, the shell would always oscillate between a minimum and maximum radius, as was shown in section III A 1. In the case of interacting bosons, we had numerically shown that the shell would oscillate only if the parameter $\alpha$ is above a certain value (see section III B).

However, in reality, since different shells evolve at different rates, as a shell is contracting, it will interact with inner shells which are expanding again after their initial contraction. When they interact, different shells will repel each other due to the quantum pressure and repel or attract each other according to their force of interaction. This would cause the density profile to depart from its initial power law shape (see, for instance, [57]). Furthermore, at the time when radius of the shell reaches its minimum, the wave function of the shell vanishes which might cause the Madelung transformation to break down (see, for instance, [58]). Thus, the shell would now have a more complicated dynamics which is not captured by the equation of motion Eq. (3.2). Nevertheless, since we know that the sphere of FDM would eventually virialize to become a halo [33], we can use the virial theorem to investigate beyond the validity of Eq. (3.2). For simplicity and because of the existence of an analytical solution, we will consider the case of non-interacting bosons in this section.

The total energy of the system is given by

$$
E_{t o t}=T+U_{Q}+U_{I}+U_{G}
$$

where, $T$ is the kinetic energy of the system, $U_{Q}$ is the energy stored in the system due to the quantum pressure, $U_{I}$ is the energy stored in the system due to the interaction and $U_{G}$ is the gravitational potential energy. When the system achieves virial equilibrium, the virial theorem states that (see, for instance, [38])

$$
2 T+2 U_{Q}+3 U_{I}+U_{G}=0 .
$$


For non-interacting bosons, the virial theorem hence implies that

$$
T+U_{Q}=-U_{G} / 2
$$

which in turn implies that the total energy of the virialized halo is given by $E_{t o t}=U_{G} / 2$.

At turn around, the energy of the system is dominated by the gravitational energy. This can be seen from the fact that the value of $\gamma$ in Eq. (3.7) is small. Thus, at turn around, $E_{t o t} \simeq U_{G}$. Using the fact that energy of the system is conserved and comparing the total energy at turn around and at virialization, one obtains, $L\left(t_{v i r}\right)=L\left(t_{t a}\right) / 2$, where we have used the expression for gravitational potential energy to be

$$
U_{G}=\frac{\mathrm{G} M^{2}}{L(t)} \frac{(3-\gamma)^{2}}{3(5-2 \gamma)}
$$

Using the expression for radius of the shell at turn around and at virialization we will now compute the overdensity of the system at turn around and virialization in the full and the linear theory. At turn around, the overdensity in the full theory is computed using Eq. (3.9) and is given by,

$$
1+\bar{\delta}_{t a}=\frac{9}{2} \frac{(\pi-e \sin (\pi))^{2}}{(1-e \cos (\pi))^{3}}=\frac{9}{2} \frac{\pi^{2}}{(1+e)^{3}} .
$$

At virialization, the overdensity is given by

$$
1+\bar{\delta}_{v i r}=\frac{9 \mathrm{G} M}{2} \frac{t_{v i r}^{2}}{L\left(t_{v i r}\right)^{3}} .
$$

Using the expressions for the radius of the shell at turn around, and hence computing virial radius, $L_{v i r}$, using $L_{v i r}=L_{t a} / 2$, one can compute the overdensity after virialization at $t_{v i r}=t(2 \pi)$ as

$$
\begin{aligned}
1+\bar{\delta}_{v i r} & =\frac{9 \mathrm{G} M}{2}\left[\left(\frac{A^{3}}{\mathrm{G} M}\right)^{1 / 2}(2 \pi)\right]^{2} \times \frac{8}{A^{3}(1+e)^{3}} \\
& =18 \pi^{2} \frac{8}{(1+e)^{3}} .
\end{aligned}
$$

It can be verified that the averaged overdensity in the full theory matches with the CDM value in the $e \rightarrow 1$ limit.

Let us now compute the overdensity in the linear regime. As we discussed in section III A 3, the small $\vartheta$ limit of $\bar{\delta}$ exists only in the limit $e \rightarrow 1$. In that limit the linear overdensity $\bar{\delta} \propto \vartheta^{2}$ as is shown in Eq. (3.11). In order to express the linear overdensity in terms of time, we need to expand Eq. (3.5b) in the $e \rightarrow 1, \vartheta \rightarrow 0$ limit. Expanding the expression for $t(\vartheta)$ in the $e \rightarrow 1$, $\vartheta \rightarrow 0$ limit, one obtains

$$
t \simeq\left(\frac{m A^{3}}{k}\right)^{1 / 2} \frac{e \theta^{3}}{6} .
$$

Using the above expression, one could write an expression for an overdensity at an initial time $t_{i}$ corresponding to $\vartheta_{i}$ as

$$
\bar{\delta}_{i}=\frac{3 \theta_{i}^{2}}{20}=\frac{3}{20}\left[\frac{6 \pi}{e} \frac{t_{i}}{t_{t a}}\right]^{2 / 3}
$$




\begin{tabular}{|c|c|c|}
\hline$t$ & Linear theory & Full theory \\
\hline$t_{t a}$ & $\frac{1.06}{e^{2 / 3}}$ & $\frac{9}{2} \frac{\pi^{2}}{(1+e)^{3}}-1$ \\
\hline$t_{v i r}$ & $\frac{1.69}{e^{2 / 3}}$ & $18 \pi^{2} \frac{8}{(1+e)^{3}}-1$ \\
\hline
\end{tabular}

TABLE I. The averaged overdensity, $\bar{\delta}$, in the linear and the full theory at turn around and virialization. It can be verified that the averaged overdensity matches with the CDM result in the $e \rightarrow 1$ limit.

In an EdS universe, since $\delta \propto a$ in the linear regime, one could write an expression for $\delta(t)$ as

$$
\begin{aligned}
\bar{\delta} & \propto \bar{\delta}_{i} \frac{a}{a_{i}} \\
& =\frac{3}{20}\left(\frac{6 \pi}{e}\right)^{2 / 3}\left(\frac{t}{t_{t a}}\right)^{2 / 3} .
\end{aligned}
$$

If we use the linear theory to compute the overdensity at turn around, one obtains,

$$
\bar{\delta}\left(t_{t a}\right) \simeq \frac{3}{20}\left(\frac{6 \pi}{e}\right)^{2 / 3}=\frac{1.06}{e^{2 / 3}} .
$$

Upon using the linear theory to compute the overdensity after virialization, i.e. at $t_{v i r}=t(2 \pi)$, we get

$$
\bar{\delta}\left(t_{v i r}\right) \simeq \frac{3}{20}\left(\frac{12 \pi}{e}\right)^{2 / 3}=\frac{1.69}{e^{2 / 3}} .
$$

The results obtained in this section have been summarized in Table I. It shows that, in this model, when the linear averaged overdensity, reaches a critical value, $\bar{\delta} \simeq 1.69 / e^{2 / 3} \simeq 1.69$, the overdense region would have virialized to form a halo.

\section{DISCUSSION}

FDM is a compelling model for dark matter. The quantum nature of FDM which gets manifested at kilo parsec scales is capable of resolving the small scale issues that has been ailing CDM. FDM halo can be described as a self-gravitating BEC and hence is governed by the GPP equations (2.1). Numerical simulations [33] indicate that at large scales the structure formed in FDM is similar to that in CDM. High resolution simulations [33] show the existence of standing waves of dark matter which evolves in to solitonic cores at the center of the halo. As they accrete more matter, the solitonic core grows and are surrounded by virialized halos with fine-scale, large-amplitude fringes. The surrounding halos are supported against gravity by quantum and turbulent pressure and hence fluctuates in density and velocity.

With the goal of gaining analytical insights in to the evolution of an FDM halo, we investigated the gravitational collapse of a spherical shell containing an overdense region of FDM. We studied the system in its hydrodynamical form, i.e. as a fluid with density $\rho$ and velocity $\vec{u}$ evolving under the effect of opposing forces of Newtonian gravity and quantum pressure. In an expanding 
universe, we computed the equation of motion governing a spherical shell Eq. (2.18). Assuming a spherically symmetric power law profile Eq. (3.1) for the overdense region, we derived an expression for the time evolution of the spherical shell Eqs. (3.5) for the case of non-interacting bosons. The correctness of the analytical solution was further established by comparing it with numerical solution. Using the analytical expressions, we arrived at an expression for the averaged overdensity enclosed by the spherical shell Eq. (3.9). Further, we numerically evaluated the evolution of a shell in the presence of interaction and compared it with the analytical expressions evaluated for the case of non-interacting bosons.

We find that, as in the case of CDM, in the beginning, the spherical shell of FDM enclosing the overdense region expands along with the Hubble flow and eventually turns around and begin to collapse. However, contrary to the case of CDM, due to the existence of quantum pressure, the FDM spherical shell eventually gets repelled and starts expanding again. A similar behaviour can be seen by looking at the expression of overdensity as well. We can see from Eq. (3.9) that the overdensity remains finite and fluctuates with time. The expression for overdensity also has the nice feature that it reproduces the CDM result in the $\hbar / m \rightarrow 0$ limit. We further studied the initial linear evolution of the overdensity. It was found that, in this model, for an overdensity, a valid small $\vartheta$ limit exists only in the $e \rightarrow 1$ limit. In the presence of interactions, the force due to interaction works along with the quantum pressure if the interaction is repulsive while acts against quantum pressure if it is attractive. We found that, for the parameters of interest, the spherical shell would oscillate in the case of attractive interaction, only if $\left|a_{s}\right|<<\bar{a}_{s}$. On the other hand, for repulsive interaction, the shell would oscillate with a larger maximum and minimum radius for larger values of $a_{s}$. We also found that, for the parameters that we have considered, which correspond to the numbers arrived for a dwarf spheroidal [33], the analytical expression for the shell is a good approximation at large to medium scales.

As the shell contracts, it will interact with inner shells and the dynamics of the shell would be more complicated than the one captured by Eq. (3.5). In reality, as was shown in [33], the spherical overdense region would eventually virialize to form a halo. Hence, the solutions discussed above, though captures some of the effects of various forces at play, will not be valid through out the evolution. However, we can investigate beyond the validity of the solutions Eq. (3.5), by making use of virial theorem. In section IV, we used the virial theorem to compute the overdensity after virialization in the linear and in the full theory (see Table I). In this model, as in the case of CDM [56], we find the critical density at which the overdensity virializes to a halo to be $\bar{\delta}_{c} \simeq$ $1.69 / e^{2 / 3} \simeq 1.69$. Finally, from the simulations performed for Fornax dwarf spheroidal, see Fig. 1, our computations shows that the virialized halo would have a radius of $L_{\text {vir }} \simeq L_{\max } / 2=.95 \mathrm{pc}$.

We shall conclude this article by discussing some of the subtleties involved in the calculation and some interesting aspects that need to be further investigated. First of all, even though this study was motivated by the possibility of FDM being a viable dark matter candidate, the analytical calculations performed in this paper hold for any non-interacting BEC collapsing under the effect of gravity. Secondly, in the case of CDM, as the shell is contracting it will cross the shells which is expanding after their first in fall. In the case of FDM, however, when two shells come close to each other there will be repulsion due to the quantum pressure and hence the dynamics near shell crossing would be more involved than in CDM. Finally, in this work we have used the hydrodynamic description to model the system. It is not clear how well the hydrodynamic description captures the physics underlying the GPP equations (see, for instance, [57-59]). Hence, it would be interesting to investigate the regime close to the "shell crossing" in more detail. 


\section{ACKNOWLEDGEMENTS}

The author would like to thank T. Padmanabhan, Aseem Paranjape and L. Sriramkumar for extensive discussions and comments. He would also like to thank the referee for useful suggestions.

[1] N. Aghanim et al. (Planck Collaboration), "Planck 2018 results. VI. Cosmological parameters," (2018), arXiv:1807.06209 [astro-ph.CO].

[2] James S. Bullock and Michael Boylan-Kolchin, "Small-Scale Challenges to the $\Lambda$ CDM Paradigm," Ann. Rev. Astron. Astrophys. 55, 343-387 (2017), arXiv:1707.04256 [astro-ph.CO].

[3] Julio F. Navarro, Carlos S. Frenk, and Simon D. M. White, "A Universal density profile from hierarchical clustering," Astrophys. J. 490, 493-508 (1997), arXiv:astro-ph/9611107 [astro-ph].

[4] W. J. G. de Blok, Stacy S. McGaugh, Albert Bosma, and Vera C. Rubin, "Mass density profiles of LSB galaxies," Astrophys. J. 552, L23-L26 (2001), arXiv:astro-ph/0103102 [astro-ph].

[5] W. J. G. de Blok, "Halo mass profiles and low surface brightness galaxies rotation curves," Astrophys. J. 634, 227-238 (2005), arXiv:astro-ph/0506753 [astro-ph].

[6] Se-Heon Oh, W. J. G. de Blok, Fabian Walter, Elias Brinks, and Robert C. Kennicutt, Jr, "Highresolution dark matter density profiles of THINGS dwarf galaxies: Correcting for non-circular motions," Astron. J. 136, 2761 (2008), arXiv:0810.2119 [astro-ph].

[7] W. J. G. de Blok, "The Core-Cusp Problem," Advances in Astronomy 2010, 789293 (2010), arXiv:0910.3538.

[8] Anatoly A. Klypin, Andrey V. Kravtsov, Octavio Valenzuela, and Francisco Prada, "Where are the missing Galactic satellites?" Astrophys. J. 522, 82-92 (1999), arXiv:astro-ph/9901240 [astro-ph].

[9] S. Garrison-Kimmel, P. F. Hopkins, A. Wetzel, J. S. Bullock, M. Boylan-Kolchin, D. Keres, C.-A. Faucher-Giguere, K. El-Badry, A. Lamberts, E. Quataert, and R. Sanderson, "The Local Group on FIRE: Dwarf galaxy populations across a suite of hydrodynamic simulations," ArXiv e-prints (2018), arXiv:1806.04143.

[10] Y. P. Jing, "Warm Dark Matter Model of Galaxy Formation," Modern Physics Letters A 16, 1795-1800 (2001), astro-ph/0201146.

[11] P. Colin, O. Valenzuela, and V. Avila-Reese, "On the Structure of Dark Matter Halos at the Damping Scale of the Power Spectrum with and without Relict Velocities," Astrophys. J. 673, 203-214 (2008), arXiv:0709.4027 [astro-ph].

[12] Jesper Sommer-Larsen and Alexandre Dolgov, "Formation of disk galaxies: warm dark matter and the angular momentum problem," Astrophys. J. 551, 608-623 (2001), arXiv:astro-ph/9912166 [astro-ph].

[13] Vijay K. Narayanan, David N. Spergel, Romeel Dave, and Chung-Pei Ma, "Constraints on the mass of warm dark matter particles and the shape of the linear power spectrum from the Ly $\alpha$ forest," Astrophys. J. 543, L103-L106 (2000), arXiv:astro-ph/0005095 [astro-ph].

[14] Paolo Salucci and Nicola Turini, "Evidences for Collisional Dark Matter In Galaxies?" (2017), arXiv:1707.01059 [astro-ph.CO].

[15] David N. Spergel and Paul J. Steinhardt, "Observational evidence for selfinteracting cold dark matter," Phys. Rev. Lett. 84, 3760-3763 (2000), arXiv:astro-ph/9909386 [astro-ph].

[16] Naoki Yoshida, Volker Springel, Simon D. M. White, and Giuseppe Tormen, "Collisional dark matter and the structure of dark halos," Astrophys. J. 535, L103 (2000), arXiv:astro-ph/0002362 [astro-ph].

[17] Wayne Hu, Rennan Barkana, and Andrei Gruzinov, "Fuzzy cold dark matter: The wave properties of ultralight particles," Phys. Rev. Lett. 85, 1158-1161 (2000).

[18] Pierre Sikivie, "Axion Cosmology," Axions: Theory, cosmology, and experimental searches. Proceedings, 1st Joint ILIAS-CERN-CAST axion training, Geneva, Switzerland, November 30-December 2, 2005, Lect. Notes Phys. 741, 19-50 (2008), arXiv:astro-ph/0610440 [astro-ph].

[19] David J. E. Marsh, "Axion Cosmology," Phys. Rept. 643, 1-79 (2016), arXiv:1510.07633 [astro-ph.CO].

[20] William H. Press, Barbara S. Ryden, and David N. Spergel, "Single mechanism for generating largescale structure and providing dark missing matter," Phys. Rev. Lett. 64, 1084-1087 (1990).

[21] Sang-Jin Sin, "Late time cosmological phase transition and galactic halo as Bose liquid," Phys. Rev. D50, 3650-3654 (1994), arXiv:hep-ph/9205208 [hep-ph]. 
[22] Varun Sahni and Li-Min Wang, "A New cosmological model of quintessence and dark matter," Phys. Rev. D62, 103517 (2000), arXiv:astro-ph/9910097 [astro-ph].

[23] David J. E. Marsh and Joe Silk, "A Model For Halo Formation With Axion Mixed Dark Matter," Mon. Not. Roy. Astron. Soc. 437, 2652-2663 (2014), arXiv:1307.1705 [astro-ph.CO].

[24] Lam Hui, Jeremiah P. Ostriker, Scott Tremaine, and Edward Witten, "Ultralight scalars as cosmological dark matter," Phys. Rev. D95, 043541 (2017), arXiv:1610.08297 [astro-ph.CO].

[25] Asimina Arvanitaki, Savas Dimopoulos, Sergei Dubovsky, Nemanja Kaloper, and John March-Russell, "String Axiverse," Phys. Rev. D81, 123530 (2010), arXiv:0905.4720 [hep-th].

[26] Jihn E. Kim and David J. E. Marsh, "An ultralight pseudoscalar boson," Phys. Rev. D93, 025027 (2016), arXiv:1510.01701 [hep-ph].

[27] Peter W. Graham and Surjeet Rajendran, "New Observables for Direct Detection of Axion Dark Matter," Phys. Rev. D88, 035023 (2013), arXiv:1306.6088 [hep-ph].

[28] Y. V. Stadnik and V. V. Flambaum, "Searching for dark matter and variation of fundamental constants with laser and maser interferometry," Phys. Rev. Lett. 114, 161301 (2015), arXiv:1412.7801 [hep-ph].

[29] Y. V. Stadnik and V. V. Flambaum, "Can dark matter induce cosmological evolution of the fundamental constants of Nature?" Phys. Rev. Lett. 115, 201301 (2015), arXiv:1503.08540 [astro-ph.CO].

[30] C. Abel et al., "Search for Axionlike Dark Matter through Nuclear Spin Precession in Electric and Magnetic Fields," Phys. Rev. X7, 041034 (2017), arXiv:1708.06367 [hep-ph].

[31] Aurlien Hees, Olivier Minazzoli, Etienne Savalle, Yevgeny V. Stadnik, and Peter Wolf, "Violation of the equivalence principle from light scalar dark matter," Phys. Rev. D98, 064051 (2018), arXiv:1807.04512 [gr-qc].

[32] Tak-Pong Woo and Tzihong Chiueh, "High-Resolution Simulation on Structure Formation with Extremely Light Bosonic Dark Matter," Astrophys. J. 697, 850-861 (2009), arXiv:0806.0232 [astro-ph].

[33] Hsi-Yu Schive, Tzihong Chiueh, and Tom Broadhurst, "Cosmic Structure as the Quantum Interference of a Coherent Dark Wave," Nature Phys. 10, 496-499 (2014), arXiv:1406.6586 [astro-ph.GA].

[34] M. Nori and M. Baldi, "AX-GADGET: a new code for cosmological simulations of Fuzzy Dark Matter and Axion models," Mon. Not. Roy. Astron. Soc. 478, 3935 (2018), arXiv:1801.08144 [astro-ph.CO].

[35] Jan Veltmaat, Jens C. Niemeyer, and Bodo Schwabe, "Formation and structure of ultralight bosonic dark matter halos," Phys. Rev. D98, 043509 (2018), arXiv:1804.09647 [astro-ph.CO].

[36] Remo Ruffini and Silvano Bonazzola, "Systems of self-gravitating particles in general relativity and the concept of an equation of state," Phys. Rev. 187, 1767-1783 (1969).

[37] C. G. Boehmer and T. Harko, "Can dark matter be a Bose-Einstein condensate?" JCAP 0706, 025 (2007), arXiv:0705.4158 [astro-ph].

[38] P.-H. Chavanis, "Mass-radius relation of Newtonian self-gravitating Bose-Einstein condensates with short-range interactions. I. Analytical results," Phys. Rev. D 84, 043531 (2011), arXiv:1103.2050.

[39] P.-H. Chavanis and L. Delfini, "Mass-radius relation of Newtonian self-gravitating Bose-Einstein condensates with short-range interactions. II. Numerical results," Phys. Rev. D 84, 043532 (2011), arXiv:1103.2054 [astro-ph.CO].

[40] Pierre-Henri Chavanis and Tiberiu Harko, "Bose-Einstein Condensate general relativistic stars," Phys. Rev. D86, 064011 (2012), arXiv:1108.3986 [astro-ph.SR].

[41] Tiberiu Harko, "Gravitational collapse of Bose-Einstein condensate dark matter halos," Phys. Rev. D89, 084040 (2014), arXiv:1403.3358 [gr-qc].

[42] Pierre-Henri Chavanis, "Collapse of a self-gravitating Bose-Einstein condensate with attractive selfinteraction," Phys. Rev. D94, 083007 (2016), arXiv:1604.05904 [astro-ph.CO].

[43] Rodolfo C. de Freitas and Hermano Velten, "Nonlinear clustering during the BEC dark matter phase transition," Eur. Phys. J. C75, 597 (2015), arXiv:1503.01877 [astro-ph.CO].

[44] Joshua Eby, Madelyn Leembruggen, Peter Suranyi, and L. C. R. Wijewardhana, "Collapse of Axion Stars," JHEP 12, 066 (2016), arXiv:1608.06911 [astro-ph.CO].

[45] Enrico D. Schiappacasse and Mark P. Hertzberg, "Analysis of Dark Matter Axion Clumps with Spherical Symmetry," JCAP 1801, 037 (2018), [Erratum: JCAP1803,no.03,E01(2018)], arXiv:1710.04729 [hep$\mathrm{ph}$.

[46] M. I. Khlopov, B. A. Malomed, and I. B. Zeldovich, "Gravitational instability of scalar fields and formation of primordial black holes," Mon. Not. Roy. Astron. Soc. 215, 575-589 (1985).

[47] A. S. Sakharov and M. Y. Khlopov, "The nonhomogeneity problem for the primordial axion field," Physics of Atomic Nuclei 57, 485-487 (1994). 
[48] A. S. Sakharov, D. D. Sokoloff, and M. Yu. Khlopov, "Large scale modulation of the distribution of coherent oscillations of a primordial axion field in the universe," Phys. Atom. Nucl. 59, 1005-1010 (1996), [Yad. Fiz.59N6,1050(1996)].

[49] M. Yu. Khlopov, A. S. Sakharov, and D. D. Sokoloff, "The large scale modulation of the density distribution in standard axionic CDM and its cosmological and physical impact," in 2nd International Workshop on Birth of the Universe and Fundamental Physics Rome, Italy, May 19-24, 1997 (1998) arXiv:hep-ph/9812286 [hep-ph].

[50] J. E. Gunn and J. R. Gott, III, "On the Infall of Matter Into Clusters of Galaxies and Some Effects on Their Evolution," Astrophys. J. 176, 1 (1972).

[51] Franco Dalfovo, Stefano Giorgini, Lev P. Pitaevskii, and Sandro Stringari, "Theory of Bose-Einstein condensation in trapped gases," Rev. Mod. Phys. 71, 463-512 (1999).

[52] Chavanis, P. H., "Growth of perturbations in an expanding universe with bose-einstein condensate dark matter," A\&A 537, A127 (2012).

[53] E. Madelung, "Quantentheorie in hydrodynamischer form," Zeitschrift für Physik 40, 322-326 (1927).

[54] H. Goldstein, Classical mechanics, 2nd ed. (Narosa Publishing House, 6 Community Centre, Panchsheel Park, New Delhi-110 017, India, 1998).

[55] Wolfram Research, Inc., "Mathematica, Version 8.0," Champaign, IL, 2010.

[56] T. Padmanabhan, Structure formation in the universe (Cambridge University Press, University Printing House, Shaftesbury Road, Cambridge, CB2 8BS, United Kingdom, 1993).

[57] Michael Kopp, Kyriakos Vattis, and Constantinos Skordis, "Solving the Vlasov equation in two spatial dimensions with the Schrödinger method," Phys. Rev. D96, 123532 (2017), arXiv:1711.00140 [astroph.CO].

[58] Cora Uhlemann, Michael Kopp, and Thomas Haugg, "Schrödinger method as $N$-body double and UV completion of dust," Phys. Rev. D90, 023517 (2014), arXiv:1403.5567 [astro-ph.CO].

[59] Philip Mocz, Lachlan Lancaster, Anastasia Fialkov, Fernando Becerra, and Pierre-Henri Chavanis, "Schrödinger-PoissonVlasov-Poisson correspondence," Phys. Rev. D97, 083519 (2018), arXiv:1801.03507 [astro-ph.CO]. 